\title{
Buprenorphin verkürzt die Behandlungszeit beim neonatalen Abstinenzsyndrom
}

Hintergrund: Die aktuelle pharmakologische Therapie des neonatalen Drogenentzugs mit Morphin ist mit einer langwierigen Behandlung und somit Krankenhausaufenthalt verbunden. Buprenorphin ist in dieser Indikation möglicherweise wirksamer als Morphin.

Patienten und Methodik: Die monozentrische, randomisierte kontrollierte Doppelblindstudie schloss 63 reife Neugeborene (Geburt frühestens in der 37. Schwangerschaftswoche) ein, die in utero Opioiden ausgesetzt waren und nach der Geburt einen Entzug zeigten. Sie wurden randomisiert einer Behandlung mit sublingualem Buprenorphin oder peroralem Morphin zugeteilt. Neugeborene, bei denen die Entzugssymptome mit Maximaldosen der Opioide nicht be-

Kraft WK, Adeniyi-Jones SC, Chervoneva I et al. Buprenorphine for the treatment of the neonatal abstinence syndrome. N Engl J Med 2017; 376: 2341 -8 herrscht werden konnten, erhielten zusätzlich Phenobarbital. Der primäre Studienendpunkt war die Dauer der bis zum Symptomrückgang notwendigen Behandlung.
Sekundäre Endpunkte waren die Dauer des Krankenhausaufenthaltes und der Anteil der Neugeborenen, die eine zusätzliche Behandlung mit Phenobarbital benötigten. Ein weiterer sekundärer Endpunkt war die Sicherheit der Medikation.

Ergebnisse: Die mittlere Behandlungsdauer war signifikant geringer mit Buprenorphin als mit Morphin (15 vs. 28 Tage, $\mathrm{p}<$ 0,001), ebenso war die Dauer des Krankenhausaufenthaltes kürzer (21 vs. 33 Tage, $p<0,001)$. Phenobarbital wurde bei fünf von 33 Kindern (15\%) in der Buprenorphin- und bei sieben von 30 Kindern (23\%) in der Morphin-Gruppe zusätzlich gegeben ( $p$ $=0,36)$. Nebenwirkungen wurde gleich häufig in beiden Gruppen beobachtet.

Schlussfolgerungen: Bei Kindern mit neonatalem Drogenentzugssyndrom führte die Behandlung mit sublingualem $\mathrm{Bu}$ prenorphin zu einer kürzeren Behandlungszeit und zu einem kürzeren Krankenhausaufenthalt als die Behandlung mit oralem Morphin. Die Nebenwirkungsraten waren in den beiden Gruppen nicht unterschiedlich.

\section{- Kommentar von Rolf L. Schlößer, Frankfurt am Main}

\section{Eine Leitlinie wäre sinnvoll}

Unter neonatalem Abstinenzsyndrom versteht man die Entzugssymptomatik des Neugeborenen einer Mutter, die in der Schwangerschaft regelmäßig Drogen konsumiert hat. Es ist gekennzeichnet durch neurologische Symptome bis hin zu Krampfanfällen, metabolischen, vegetativen und respiratorischen Störungen sowie gastrointestinalen Problemen. Obwohl das neonatale Abstinenzsyndrom seit vielen Jahren gut untersucht ist [1], fehlen immer noch evidenzbasierte Erkenntnisse in der pharmakologischen Behandlung des Entzugs. Bislang werden sehr unterschiedliche Opiode, Sedativa oder andere zentral wirksame Medikamente wie Clonidin allein oder in Kombination eingesetzt [2, 3]. Offenbar spielt ein festgelegter lokaler Algorithmus überhaupt in der Behandlung der Patienten eine zentrale Rolle [4]. Die vorliegende Arbeit ist eine der wenigen randomisiert-kontrollierten Studien (Jadad-Score 4), die verblindet zwei Therapiestrategien vergleicht - und zwar sublinguales Buprenorphin mit oral verabreichtem Morphin. Eine placebokontrollierte Studie wäre nicht zu rechtfertigen. Das primäre Endziel war - wie in vielen Fallkontrollstudien - die Dauer der notwendigen Behandlung.

Die Länge des Krankenhausaufenthaltes und ein zusätzlicher Behandlungsbedarf mit Phenobarbital, um die Symptome zu beherrschen, waren sekundäre Endpunkte. Die Setzung solcher Endpunkte ist durchaus üblich und sinnvoll, auch wenn die psychomotorische Entwicklung der Kinder nach Ent- zug und/oder Pharmakotherapie interessanter wäre [5]. Außerdem zeigt die klinische Realität, dass der stationäre Aufenthalt eines Kindes einer drogenabhängigen Mutter von psychosozialen Aspekten determiniert wird.

Ziel weiterer randomisierter, kontrollierter Studien sollte es sein, evidenzbasierte Erkenntnisse in einer Leitlinie zusammenzufassen.
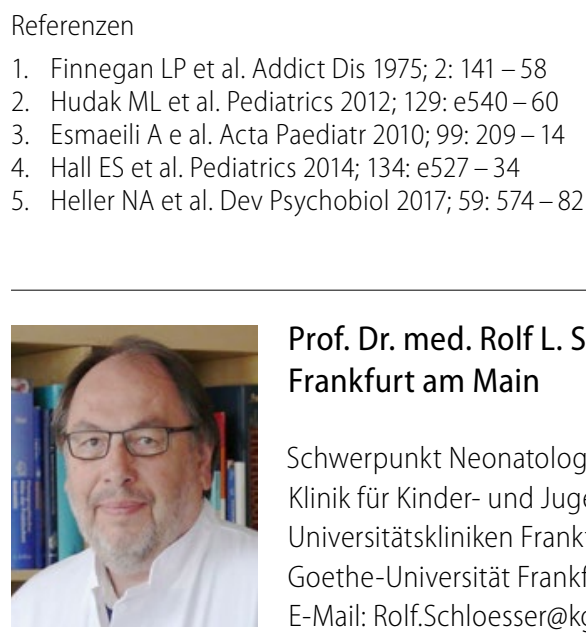

Prof. Dr. med. Rolf L. Schlößer, Frankfurt am Main

Schwerpunkt Neonatologie, Klinik für Kinder- und Jugendmedizin, Universitätskliniken Frankfurt, Goethe-Universität Frankfurt am Main E-Mail:Rolf.Schloesser@kgu.de 